Draft Version July 29, 2021

Preprint typeset using $\mathrm{IAT}_{\mathrm{E}} \mathrm{X}$ style emulateapj v. 12/16/11

\title{
SENSITIVITY OF COSMOLOGICAL PARAMETER ESTIMATION TO NONLINEAR PRESCRIPTION FROM GALAXY CLUSTERING
}

\author{
SARAh Safi, Marzieh Farhang \\ Department of Physics, Shahid Beheshti University, 1983969411, Tehran Iran
}

Draft version July 29, 2021

\begin{abstract}
Next generation large scale surveys will probe the nonlinear regime with high resolution. Making viable cosmological inferences based on these observations requires accurate theoretical modeling of the mildly nonlinear regime. In this work we investigate the sensitivity of cosmological parameter measurements from future probes of galaxy clustering to the choice of nonlinear prescription up to $k_{\max }=0.3 \mathrm{~h} \mathrm{Mpc}^{-1}$. In particular, we calculate the induced parameter bias when the mildly nonlinear regime is modeled by the Halofit fitting scheme. We find significant $(\sim 5 \sigma)$ bias for some parameters with a future Euclid-like survey. We also explore the contribution of different scales to the parameter estimation for different observational setups and cosmological scenarios, compared for the two nonlinear prescriptions of Halofit and EFTofLSS. We include in the analysis the free parameters of the nonlinear theory and a blind parametrization for the galaxy bias. We find that marginalization over these nuisance parameters significantly boosts the errors of the standard cosmological parameters. This renders the differences in the predictions of the various nonlinear prescriptions less effective when transferred to the parameter space. More accurate modeling of these nuisance parameters would therefore greatly enhance the cosmological gain from the mildly nonlinear regime.
\end{abstract}

Subject headings: mildly nonlinear regime - cosmology; theory - large scale structure

\section{INTRODUCTION}

Future large scale surveys, such as Euclid (Laureijs et al. 2011) and the square kilometer array (SKA) (Santos et al. 2015), will probe the mildly nonlinear (MNL afterwards) regime of structure formation with unprecedented accuracy. This high accuracy would translate into tight constraints on cosmological parameters. A large amount of study has gone into exploring this regime for making cosmological inferences in different frameworks (see, e.g., Martinelli et al. 2011; Majerotto et al. 2012; Di Porto et al. 2012; Wang 2012; de Putter et al. 2013; Bull 2016; Sartoris et al. 2016; Gleyzes et al. 2016; Blanchard et al. 2019; Casas et al. 2017; Sprenger et al. 2019, among many others).

On the other hand, the analysis of data from these high-resolution experiments calls for accurate theoretical modeling that reliably describes the MNL regime for optimal extraction of the available cosmological information. This regime has been investigated perturbatively by different approaches including the SPT (Bernardeau et al. 2002; Carlson et al. 2009), the RPT (Crocce \& Scoccimarro 2006), the RegPT(Taruya et al. 2012) and the EFTofLSS (Carrasco et al. 2012). For a list of theoretical methods and the scales of the validity of their predictions see Carlson et al. (2009).

The effective field theory of large scale structure, abbreviated as the EFTofLSS, is the main nonlinear analytical framework used in this work. It provides a convergent perturbation theory and is claimed to be more successful compared to various alternatives (Baumann et al. 2012; Carrasco et al. 2012, 2014). This theory generalizes the SPT by effectively taking into account the impact of short modes on large scales through the introduction of

Corresponding author email: m_farhang@sbu.ac.ir certain free coefficients to be measured by data (Carrasco et al. 2012, 2014). The EFTofLSS is so far developed at the two- and three-loop order (Carrasco et al. 2014; Baldauf et al. 2015b; Konstandin et al. 2019) with various modifications such as bias and baryonic effects taken into account (Senatore 2015; Mirbabayi et al. 2015; Assassi et al. 2014; Angulo et al. 2015a; Lewandowski et al. 2015). Various predictions of the theory have also been compared to simulations, including the dark matter density power spectrum (Carrasco et al. 2012; Senatore \& Zaldarriaga 2015; Carrasco et al. 2014), bispectrum (Angulo et al. 2015b; Baldauf et al. 2015a), dark matter momentum power spectrum (Senatore \& Zaldarriaga 2015) and the dark matter power spectrum in redshift space (Senatore \& Zaldarriaga 2014). Recently, the EFTofLSS has been applied to the analysis of the BAO data in order to examine the extent to which this theory can provide a theoretical template in cosmological parameter estimation (D'Amico et al. 2020a; Colas et al. 2020; Nishimichi et al. 2020; D'Amico et al. 2020b,c).

In this work we study the impact of the prescription for the MNL regime on cosmological parameter estimation from the galaxy clustering probe of Euclid- and SKAlike surveys. (In a recent work, Martinelli et al. (2020) studied a similar problem but for the Euclid weak lensing probe and with different nonlinear prescriptions.) We investigate how the inaccuracies in the nonlinear prescription lead to biases in the parameters. We also explore the available information in these regimes to constrain parameters in a couple of different cosmological scenarios. The impact of marginalization over parameters describing the galaxy bias and the free parameters of the nonlinear theory is also considered.

The rest of this paper is organized as follows. In Section 2 we investigate the induced bias in the parameter 
estimations due to improper choice of nonlinear prescription and in Section 3 we explore the cosmological information available for parameter measurements in the MNL regime, under different assumptions for experimental specifications and cosmological and analysis framework. We conclude in Section 4.

\section{NONLINEAR PRESCRIPTION BIAS IN PARAMETER ESTIMATION}

The accuracy and robustness of the nonlinear recipe of structure formation is of particular importance in making forecasts and analyzing the high-resolution data of future surveys. Here we investigate the insufficiencies of a particular prescription, the Halofit model (HF afterwards), in describing its impact on cosmological inferences. HF is a commonly used recipe for modeling the matter power spectrum in the MNL regime (Smith et al. 2003; Takahashi et al. 2012) and is based on fitting formulas from the results of $N$-body simulations (Ma \& Fry 2000; Seljak 2000; Cooray \& Sheth 2002).

We quantify this effect by calculating the induced shifts in the parameter estimation for several different cosmological models. As the dataset we use power spectrum simulations of galaxy clustering for a future Euclid-like survey. We review the assumptions used to simulate the observed galaxy power spectrum in Section 2.1 and the cosmological scenarios used for the analysis of the simulations in Section 2.2. The results are presented and discussed in Section 2.3.

\subsection{Simulations of Galaxy Clustering}

Here we use the HF precription to make predictions for a future Euclid-like large scale survey. We simulate our mock data based on the theoretical matter power spectrum from the ds14-a set of Dark Sky $N$-body simulation series (Skillman et al. 2014). The fiducial values of the cosmological parameters used in the simulations are $\left\{\Omega_{m}, \Omega_{b}, \Omega_{\Lambda}, h, n_{s}, \sigma_{8}\right\}=$ $\{0.295,0.0468,0.705,0.688,0.9676,0.8344\}$. From these power spectra, we simulate the observed power spectra of galaxy clustering (or GC) for Euclid-like specifications (the first column of Table 1).

The observed galaxy power spectrum, $P_{\mathrm{obs}}$, is given by (Seo \& Eisenstein 2007)

$$
\begin{aligned}
P_{\mathrm{obs}}(k, \mu, z) & =\frac{D_{A, \mathrm{ref}}^{2} H(z)}{D_{A}^{2} H_{\mathrm{ref}}(z)} b^{2}(z)\left(1+\beta(z) \mu^{2}\right)^{2} \\
& \times e^{-k^{2} \mu^{2}\left(\sigma_{\mathrm{r}}^{2}+\sigma_{\mathrm{v}}^{2}\right)} P(k, z)
\end{aligned}
$$

where $\mu$ is the cosine of the angle between the line of sight and the wavevector $\vec{k}, H(z)$ is the Hubble parameter at redshift $z$ and $D_{A}(z)$ represents the angular diameter distance to redshift $z$. We also have $\beta(z)=f(z) / b(z)$ where $f(z)$ represents the linear growth rate and $b(z)$ is the redshift-dependent galaxy bias. In this section we assume a known $z$-dependence for the galaxy bias, $b(z)=\sqrt{1+z}$ (Amendola et al. 2018). The coefficient $D_{A, \text { ref }}^{2} H(z) / D_{A}^{2} H_{\text {ref }}(z)$ encodes the geometrical distortions due to the Alcock-Paczynski effect (Alcock \& Paczyński 1979). The effect of redshift measurement uncertainty, $\sigma_{z}$, propagates into the comoving distance error $\sigma_{r}=\frac{c}{H(z)} \sigma_{z}$ (Wang et al. 2013). For the peculiar velocity dispersion we take $\sigma_{v}=300 \mathrm{~km} / \mathrm{s}$ (Casas et al. 2017). Table 1 presents the experimental specifications used for the GC probe in this work.

\begin{tabular}{cccc}
\hline & Euclid & SKA1 & SKA2 \\
\hline$f_{\text {sky }}$ & 0.36 & 0.12 & 0.73 \\
$\sigma_{z}$ & $0.001(1+z)$ & $0.001(1+z)$ & $0.001(1+z)$ \\
$\left\{z_{\min }, z_{\max }\right\}$ & $\{0.7,2\}$ & $\{0,0.7\}$ & $\{0.1,2\}$ \\
$\Delta z$ & 0.1 & 0.1 & 0.1 \\
\hline
\end{tabular}

TABLE 1

EUCLID, SKA1 AND SKA2 SPECIFICATIONS FOR GALAXY CLUSTERING WHERE $f_{\text {Sky }}$ IS THE SKY FRACTION, $\sigma_{z}$ STANDS FOR THE SPECTROSCOPIC REDSHIFT ERROR, $\left\{z_{\min }, z_{\max }\right\}$ REPRESENTS THE OBSERVED REDSHIFT INTERVAL AND $\Delta z$ IS THE WIDTH OF THE REDSHIFT BINS (AMENDOLA ET AL. 2018; BulL 2016).

\subsection{Models of Dark Energy}

In this work we consider three sets of cosmological scenarios, $\Lambda$ CDM, $w$ CDM with $w \neq-1$ a constant, and a dynamical dark energy model with its equation of state modeled as (Chevallier \& Polarski 2001; Linder 2003)

$$
w(a)=w_{0}+(1-a) w_{a} .
$$

Here $w_{0}$ and $w_{a}$ are constants and the deviation of $\left(w_{0}, w_{a}\right)$ from $(-1,0)$ encodes the departure from $\Lambda \mathrm{CDM}$.

\subsection{Analysis and Results}

The goal in this section is to calculate how the improper choice of the MNL prescription impacts the parameter estimation. Specifically, we calculate the induced bias in the parameter measurements compared to their fiducial values. For this purpose, we base our analysis on the publicly available code CosmoMC $^{1}$ (Lewis \& Bridle 2002; Lewis 2013) to sample the parameter space. CosmoMC uses HF to model the nonlinear regime. We map the parameter distribution measured by the GC power spectrum constructed from the Dark Sky simulations with Euclid specifications (Eq. 1). The analysis is performed at four redshift bins centered at $\{0.66,1,1.5,2\}$ and with $k_{\max }=0.3 h \mathrm{Mpc}^{-1}$, for the three cosmological models of $\Lambda \mathrm{CDM}, w \mathrm{CDM}$ and $\left(w_{0}, w_{a}\right)$ CDM.

The results of the parameter estimation are illustrated in Figure 1. The vertical lines indicate the fiducial parameter values used in the simulations. The measurements are found to be biased with respect to the fiducial values with different levels of significance. The few percent mismatch in the power spectra in the MNL regime up to $k_{\max }=0.3 \mathrm{~h} \mathrm{Mpc}^{-1}$ between the predictions of HF and $N$-body simulations (assumed to represent data here) leads to a few $\sigma$ bias in the parameter values for the GC probe of a Euclid-like survey. The two parameters $\sigma_{8}$ and $\Omega_{\mathrm{b}} h^{2}$ suffer from the largest biases, with $\sim(4-5) \sigma$ significance. This clearly demonstrates the need for very accurate modeling of this cosmologically interesting regime.

In the next section we investigate the impact of various scales in the MNL regime on parameter estimation for Euclid- and SKA-like surveys.

\footnotetext{
${ }^{1}$ https://cosmologist.info/cosmomc/
} 


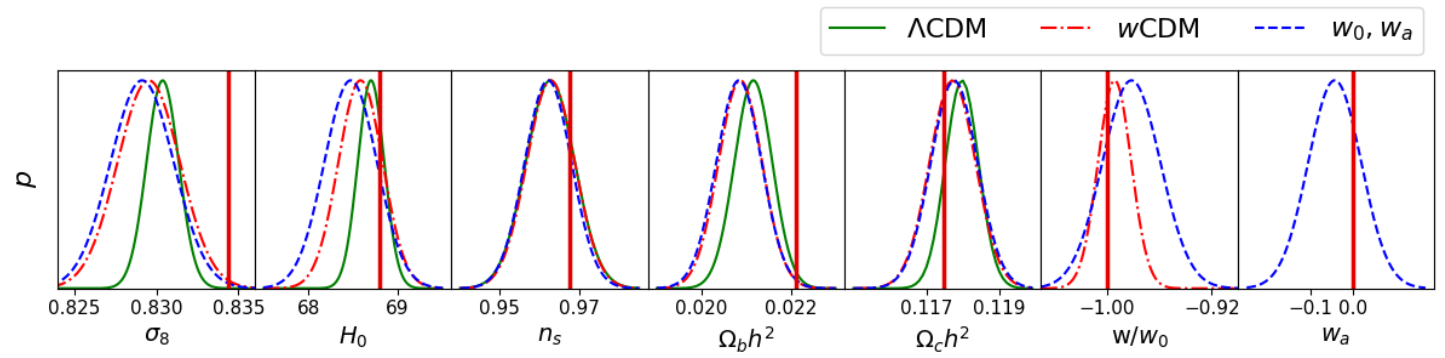

FIG. 1. - 1D marginalized parameter likelihoods for three different cosmological scenarios with $k_{\max }=0.3 \mathrm{~h} \mathrm{Mpc}^{-1}$. Vertical lines illustrate the parameter fiducial values used in the simulation. This analysis does not include any free nuisance parameters.

\section{SCALE-DEPENDENCE OF PARAMETER MEASUREMENTS}

Here we explore the information available at various scales for constraining the cosmological parameters. As the main theoretical MNL prescription, we use the EFTofLSS and compare its predictions to those from HF

\subsection{The Nonlinear Prescription}

A sound analysis of data from a Euclid-like experiment requires high, $\sim 1 \%$ precision modeling of the matter power spectrum. The accuracy of the popular HF model is claimed to be $\sim 3 \%$, rendering it unreliable for the analysis of the next generation high resolution large scale surveys. Moreover, $\mathrm{HF}$ is calibrated for $\Lambda \mathrm{CDM}$ and lacks suitable extensions for non- $\Lambda \mathrm{CDM}$ models (Amendola et al. 2018). Among the analytical approaches to investigate the MNL regime is the EFTofLSS, abbreviated as EFT in the rest of this section, which provides an effective description of the universe at large scales through integrating out short-wavelenghth perturbations. Longwavelengths are interpreted as an effective fluid characterized by few parameters such as the equation of state, the speed of sound and viscosity. The coefficients describing the short distance dynamics need to be fitted to observations or measured through $N$-body simulations (Baumann et al. 2012; Carrasco et al. 2012). The EFT predictions are claimed to have considerable improvement over the predictions from alternative models such as SPT (Carrasco et al. 2012, 2014; Foreman et al. 2016).

In the rest of this work we use EFT as the main prescription to probe into the MNL regime, along with HF for comparison. In this work we follow the parametrization of Foreman et al. (2016) to effectively encapsulate the short-wavelength modes. We refer to these parameters as sound speeds and label them generically by $c_{\mathrm{S}}$ throughout the paper. The $c_{\mathrm{s}}$ represent the three parameters, $c_{\mathrm{s}(1)}^{2}, c_{1}$ and $c_{4}$, that characterize the one and two-loop counterterms in the nonlinear corrections to the power spectrum (see Eq. 6.1 of Foreman et al. 2016). At one-loop, $c_{\mathrm{s}(1)}^{2}$ is the lowest order counterterm while $c_{1}$ and $c_{4}$ are the additional higher derivative counterterms appearing at two-loop calculations. The redshift dependence of the counterterms is parametrized with fitting functions with 12 free parameters (see Eq. 5.7 of Foreman et al. 2016).

\subsection{Analysis}

Our goal in this section is to determine the $k$ dependence of the estimated errors for various parameters as measured by the GC probe of Euclid- and SKAlike surveys. For this we use the Fisher matrix formalism to estimate the correlation matrix and thus the errors of the parameters. If the distribution of the parameters can be approximated by a Gaussian, their covariance matrix $C$ is approximated by the inverse of the Fisher matrix $\boldsymbol{C}=\boldsymbol{F}^{-1}$. In particular, the diagonal elements of the Fisher inverse correspond to the predicted errors for each parameter, $\sigma_{i}^{2}=\left(F^{-1}\right)_{i i}$. The Fisher matrix of the cosmological parameters $\boldsymbol{q}=\left\{q_{1}, \ldots, q_{N}\right\}$ for the GC at redshift $z$ can be written as (Amendola et al. 2012; Seo \& Eisenstein 2007)

$$
\begin{array}{r}
F_{i j}(z)=\frac{V_{\mathrm{sur}}}{8 \pi^{2}} \int_{-1}^{+1} d \mu \int_{k_{\min }}^{k_{\max }} d k k^{2} \frac{\partial \ln P_{\mathrm{obs}}(k, \mu, z)}{\partial q_{i}} \\
\frac{\partial \ln P_{\mathrm{obs}}(k, \mu, z)}{\partial q_{j}} \times\left[\frac{n(z) P_{\mathrm{obs}}(k, \mu, z)}{n(z) P_{\mathrm{obs}}(k, \mu, z)+1}\right]^{2}
\end{array}
$$

where $V_{\text {sur }}$ is the volume of sky covered by the galaxy survey in bins of width $\Delta z$ centered at $z$. The galaxy number density $n(z)$ is taken from Table 3 of Amendola et al. (2018) for the Euclid-like case and Tables 2 and 3 of Bull (2016) for the SKA-like cases. For the largest scales used here we take $k_{\min }=0.0079 h \mathrm{Mpc}^{-1}$. We consider two scenarios, linear with $k_{\max }=0.15 h \mathrm{Mpc}^{-1}$ and MNL with $k_{\max }=0.3 h \mathrm{Mpc}^{-1}$. The full Fisher matrix includes contributions from all redshift bins of the survey, $\boldsymbol{F}=\sum \boldsymbol{F}(z)$, with 14 and 26 bins for the Euclid and SKA-like cases respectively. See Table 1 for more experimental specifications used in this work. The fiducial values in our Fisher forecast are consistent with the Planck measurements (Aghanim et al. 2020).

The galaxy bias is assumed to have a known redshift dependence, similar to Section 2.1 for the Euclid-like case and as Tables 2 and 3 of Bull (2016) for the SKAlike cases. In parallel, we also investigate the impact of uncertainties in modeling the bias on cosmological measurements. For this purpose, in the HF-based analysis we compare the results with a second treatment of the galaxy bias where no prior assumption is imposed on the functional form of $b(z)$. Instead, we assume $b(z)$ to have a free constant value in each observation bin, and simultaneously measure it with the other cosmological parameters. The final results from this treatment are marginalized over these bias parameters. For the case of 


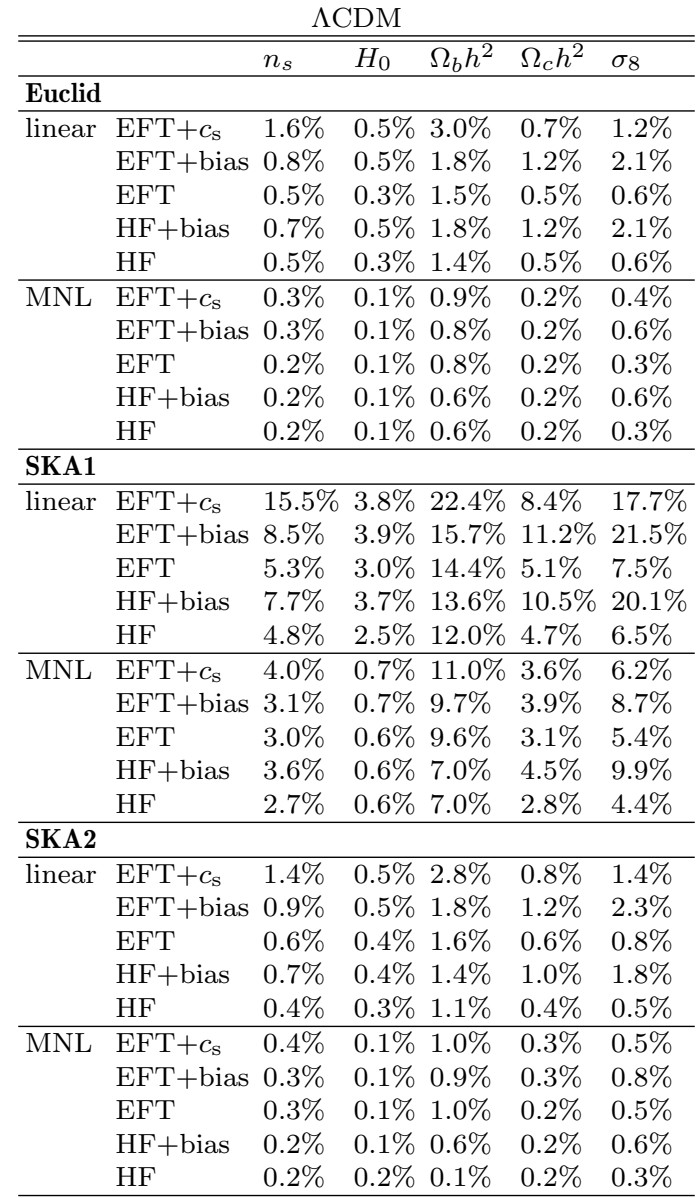

TABLE 2

THE ESTIMATED $1 \sigma$ UNCERTAINTY OF COSMOLOGICAL PARAMETERS IN $\Lambda$ CDM SCENARIO FROM GC IN THE LINEAR AND MNL REGIMES. THE PREDICTIONS ARE COMPARED FOR EFT (WITH FIXED AND MARGINALIZED $c_{\mathrm{S}}$ AND BIAS PARAMETERS) TO HF (WITH FIXED AND MARGINALIZED BIAS PARAMETERS).

EFT analysis, we also follow two paths of treating the 12 sound speed parameters of EFT, i.e., the $c_{\mathrm{s}}$, as fixed and free parameters to be marginalized over.

\subsection{Results}

Parameter errors and their $k$-dependence Tables 2 , 3 and 4 present the estimated parameter errors in the $\Lambda \mathrm{CDM}, w \mathrm{CDM}$ and $\left(w_{0}, w_{a}\right) \mathrm{CDM}$ frameworks respectively for the $\mathrm{GC}$ probe with the specifications proposed for Euclid, SKA1 and SKA2 in the two regimes of linear and MNL. The EFT results are compared for known and binned bias descriptions, as well as fixed and free $c_{\mathrm{s}}$, while the $\mathrm{HF}$ results are with known and binned bias parameters. The presented errors for all parameters are relative $1 \sigma$ errors in percentage, with respect to the parameter fiducial absolute values, expect for $w_{a}$ whose fiducial is zero and its absolute error is reported. Figure 2 illustrates the $k$-dependence of the estimated parameter errors for the $\left(w_{0}, w_{a}\right) \mathrm{CDM}$ scenario for a Euclid-like survey. The curves correspond to EFT (with fixed and marginalized $c_{\mathrm{s}}$ and bias parameters) and $\mathrm{HF}$ (with fixed and marginalized bias parameters). As we go to the MNL regime, corresponding to higher $k$ 's, the uncertainty in the cosmological parameters are

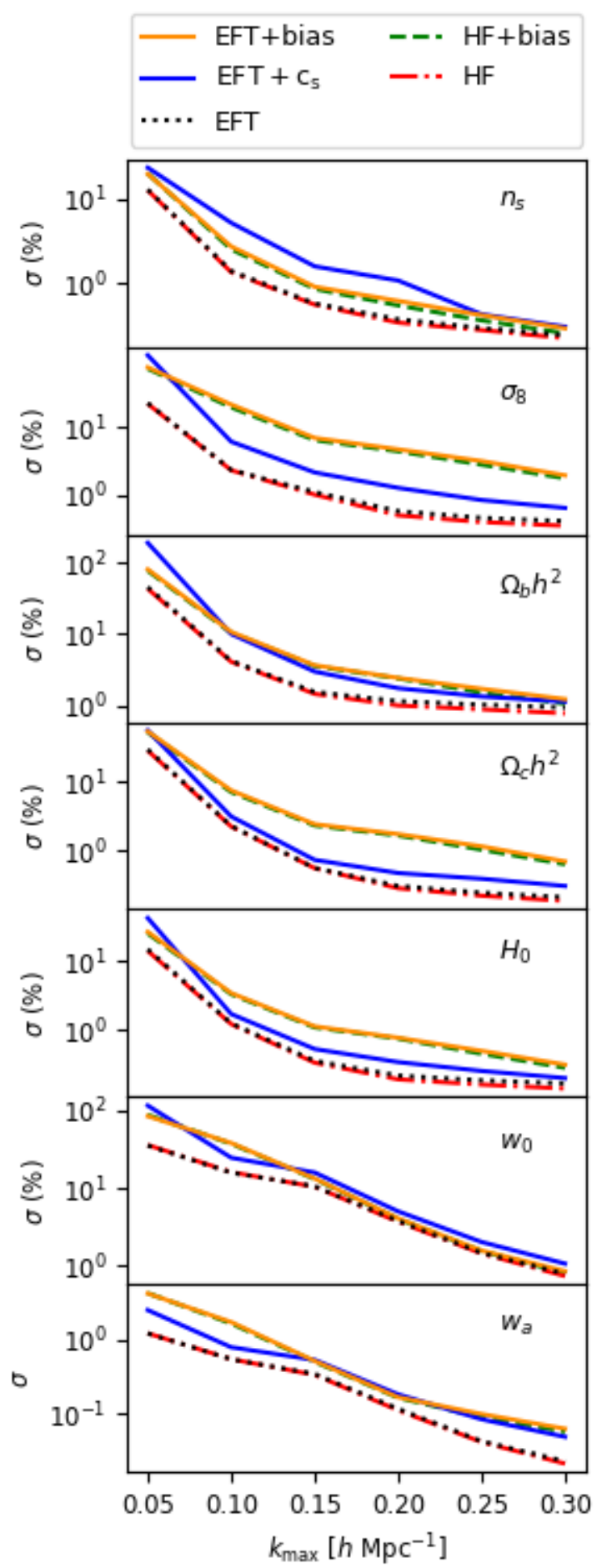

Fig. 2.- The estimated $1 \sigma$ uncertainties of cosmological parameters in the $\left(w_{0}, w_{a}\right) \mathrm{CDM}$ framework for different $k_{\max }$ for a Euclid-like survey, with the EFT and HF nonlinear recipes, assuming fixed and free $c_{\mathrm{s}}$ and bias parameters respectively. All errors are relative except for $w_{a}$. 


\begin{tabular}{|c|c|c|c|c|c|c|c|}
\hline \multicolumn{8}{|c|}{$w \mathrm{CDM}$} \\
\hline & & $n_{\mathrm{S}}$ & $\overline{H_{0}}$ & $\bar{\Omega}_{\mathrm{b} \mathrm{b}} h^{2}$ & $\bar{\Omega}_{\mathrm{c}} h^{2}$ & $\overline{\sigma_{8}}$ & $\bar{w}$ \\
\hline \multicolumn{8}{|l|}{ Euclid } \\
\hline \multirow[t]{5}{*}{ linear } & $\mathrm{EFT}+c_{\mathrm{s}}$ & $1.6 \%$ & $0.5 \%$ & $3.0 \%$ & $0.7 \%$ & $1.4 \%$ & $2.6 \%$ \\
\hline & EFT+bias & $0.9 \%$ & $1.0 \%$ & $3.2 \%$ & $2.1 \%$ & $5.3 \%$ & $5.4 \%$ \\
\hline & EFT & $0.6 \%$ & $0.4 \%$ & $1.5 \%$ & $0.5 \%$ & $0.7 \%$ & $1.2 \%$ \\
\hline & $\mathrm{HF}+\mathrm{bias}$ & $0.8 \%$ & $1.0 \%$ & $3.1 \%$ & $2.0 \%$ & $5.0 \%$ & $5.2 \%$ \\
\hline & $\mathrm{HF}$ & $0.6 \%$ & $0.3 \%$ & $1.4 \%$ & $0.5 \%$ & $0.7 \%$ & $1.2 \%$ \\
\hline \multirow[t]{5}{*}{$\overline{M N L}$} & $\mathrm{EFT}+c_{\mathrm{S}}$ & $0.3 \%$ & $0.2 \%$ & $1.1 \%$ & $0.2 \%$ & $0.4 \%$ & $0.6 \%$ \\
\hline & $\mathrm{EFT}+$ bias & $0.2 \%$ & $0.2 \%$ & $1.0 \%$ & $0.3 \%$ & $0.9 \%$ & $0.8 \%$ \\
\hline & EFT & $0.2 \%$ & $0.2 \%$ & $0.9 \%$ & $0.2 \%$ & $0.4 \%$ & $0.5 \%$ \\
\hline & $\mathrm{HF}+\mathrm{bias}$ & $0.2 \%$ & $0.2 \%$ & $0.9 \%$ & $0.3 \%$ & $1.0 \%$ & $0.8 \%$ \\
\hline & $\mathrm{HF}$ & $0.2 \%$ & $0.1 \%$ & $0.8 \%$ & $0.2 \%$ & $0.3 \%$ & $0.5 \%$ \\
\hline \multicolumn{8}{|l|}{$\overline{\text { SKA1 }}$} \\
\hline \multirow[t]{5}{*}{ linear } & $\mathrm{EFT}+c_{\mathrm{s}}$ & $15.8 \%$ & $5.3 \%$ & $27.4 \%$ & $8.4 \%$ & $23.2 \%$ & $29.2 \%$ \\
\hline & $\mathrm{EFT}+\mathrm{bias}$ & $9.1 \%$ & $9.9 \%$ & $32.8 \%$ & $21.0 \%$ & $63.4 \%$ & $50.9 \%$ \\
\hline & EFT & $6.3 \%$ & $3.7 \%$ & $16.4 \%$ & $5.2 \%$ & $10.8 \%$ & $17.6 \%$ \\
\hline & $\mathrm{HF}+\mathrm{bias}$ & $9.0 \%$ & $11.1 \%$ & $35.3 \%$ & $24.1 \%$ & $73.4 \%$ & $55.0 \%$ \\
\hline & $\mathrm{HF}$ & $5.8 \%$ & $3.2 \%$ & $14.2 \%$ & $4.7 \%$ & $11.5 \%$ & $19.7 \%$ \\
\hline \multirow[t]{5}{*}{ MNL } & $\mathrm{EFT}+c_{\mathrm{s}}$ & $4.0 \%$ & $1.1 \%$ & $11.4 \%$ & $3.8 \%$ & $7.8 \%$ & $7.3 \%$ \\
\hline & $\mathrm{EFT}+\mathrm{bias}$ & $3.1 \%$ & $1.1 \%$ & $10.2 \%$ & $4.5 \%$ & $11.3 \%$ & $7.2 \%$ \\
\hline & EFT & $3.0 \%$ & $0.8 \%$ & $9.8 \%$ & $3.2 \%$ & $6.2 \%$ & $5.0 \%$ \\
\hline & $\mathrm{HF}+\mathrm{bias}$ & $3.7 \%$ & $1.1 \%$ & $7.4 \%$ & $5.6 \%$ & $14.1 \%$ & $7.6 \%$ \\
\hline & $\mathrm{HF}$ & $2.7 \%$ & $0.8 \%$ & $7.2 \%$ & $2.9 \%$ & $5.6 \%$ & $5.3 \%$ \\
\hline \multicolumn{8}{|l|}{$\overline{\text { SKA2 }}$} \\
\hline \multirow[t]{5}{*}{ linear } & $\mathrm{EFT}+c_{\mathrm{s}}$ & $1.4 \%$ & $0.5 \%$ & $3.0 \%$ & $0.8 \%$ & $1.5 \%$ & $2.3 \%$ \\
\hline & EFT+bias & $1.0 \%$ & $1.1 \%$ & $3.5 \%$ & $2.3 \%$ & $5.7 \%$ & $4.7 \%$ \\
\hline & EFT & $0.6 \%$ & $0.4 \%$ & $1.6 \%$ & $0.6 \%$ & $0.8 \%$ & $1.1 \%$ \\
\hline & $\mathrm{HF}+\mathrm{bias}$ & $0.7 \%$ & $0.8 \%$ & $2.6 \%$ & $1.6 \%$ & $4.2 \%$ & $3.4 \%$ \\
\hline & $\mathrm{HF}$ & $0.5 \%$ & $0.3 \%$ & $1.2 \%$ & $0.4 \%$ & $0.6 \%$ & $0.8 \%$ \\
\hline \multirow[t]{5}{*}{$\overline{M N L}$} & $\mathrm{EFT}+c_{\mathrm{s}}$ & $0.4 \%$ & $0.1 \%$ & $1.1 \%$ & $0.3 \%$ & $0.6 \%$ & $0.6 \%$ \\
\hline & EFT+bias & $0.3 \%$ & $0.2 \%$ & $1.1 \%$ & $0.5 \%$ & $1.3 \%$ & $0.9 \%$ \\
\hline & $\mathrm{EFT}$ & $0.3 \%$ & $0.1 \%$ & $1.0 \%$ & $0.3 \%$ & $0.5 \%$ & $0.4 \%$ \\
\hline & $\mathrm{HF}+$ bias & $0.3 \%$ & $0.2 \%$ & $0.8 \%$ & $0.5 \%$ & $1.3 \%$ & $0.8 \%$ \\
\hline & $\mathrm{HF}$ & $0.2 \%$ & $0.1 \%$ & $0.6 \%$ & $0.2 \%$ & $0.3 \%$ & $0.3 \%$ \\
\hline
\end{tabular}

TABLE 3

Similar to Table 2 BUt for a $w$ CDM scenario. We USED the ABSOLUTE VALUE OF $w$ IN THE RELATIVE ERROR ESTIMATION.

significantly reduced indicating the large amount of information available in these small scales. In the three cosmic scenarios, and with the bias and $c_{\mathrm{s}}$ fixed, the uncertainties are very close for HF and EFT in the linear regime as expected. The tiny mismatch for some parameters, with HF slightly underestimating the error, is not surprising since nonlinearities are not fully negligible at $k \sim 0.15 h \mathrm{Mpc}^{-1}$. The inclusion of larger wavenumbers significantly improves the measurements. The estimated errors are still comparable for the two MNL recipes, with the HF-based analysis underestimating some of the uncertainties. On the other hand, if the tight prior on the bias and $c_{\mathrm{s}}$ are relaxed and they are allowed to vary, there is notable boost in the uncertainties which varies for different parameters. It is interesting to note that this boost is significant for some parameters even in the linear regime. This is induced by the correlation between the $c_{\mathrm{S}}$ and some of the cosmological parameters which enhances the errors due to lack of information in the linear regime. In the MNL regime, with more data available, the correlations partially break and the errors decrease.

Correlation matrix The inclusion of larger wavenumbers affects the parameter distribution in the parameter space and therefore their measurements not only through

\begin{tabular}{|c|c|c|c|c|c|c|c|c|}
\hline & & $n_{s}$ & $H_{0}$ & $\overline{\Omega_{b} h^{2}}$ & 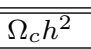 & $\sigma_{8}$ & $w_{0}$ & $w_{a}$ \\
\hline \multicolumn{9}{|l|}{ Euclid } \\
\hline \multirow[t]{5}{*}{ linear } & $\mathrm{EFT}+c_{\mathrm{s}}$ & $1.6 \%$ & $0.5 \%$ & $3.0 \%$ & $0.7 \%$ & $2.2 \%$ & $15.9 \%$ & 0.5 \\
\hline & $\mathrm{EFT}+$ bias & $0.9 \%$ & $1.1 \%$ & $3.7 \%$ & $2.4 \%$ & $7.0 \%$ & $13.3 \%$ & 0.5 \\
\hline & EFT & $0.6 \%$ & $0.4 \%$ & $1.5 \%$ & $0.5 \%$ & $1.1 \%$ & $10.6 \%$ & 0.2 \\
\hline & $\mathrm{HF}+$ bias & $0.9 \%$ & $1.1 \%$ & $3.6 \%$ & $2.3 \%$ & $6.5 \%$ & $13.1 \%$ & 0.5 \\
\hline & $\mathrm{HF}$ & $0.6 \%$ & $0.3 \%$ & $1.4 \%$ & $0.5 \%$ & $1.0 \%$ & $10.4 \%$ & 0.3 \\
\hline \multirow[t]{5}{*}{$\overline{M N L}$} & $\mathrm{EFT}+c_{\mathrm{S}}$ & $0.3 \%$ & $0.2 \%$ & $1.1 \%$ & $0.3 \%$ & $0.6 \%$ & $1.1 \%$ & $\overline{0.06}$ \\
\hline & $\mathrm{EFT}+$ bias & $0.4 \%$ & $0.2 \%$ & $1.2 \%$ & $0.5 \%$ & $1.3 \%$ & $1.3 \%$ & 0.03 \\
\hline & EFT & $0.2 \%$ & $0.2 \%$ & $0.9 \%$ & $0.2 \%$ & $0.4 \%$ & $0.8 \%$ & 0.01 \\
\hline & $\mathrm{HF}+$ bias & $0.3 \%$ & $0.3 \%$ & $1.1 \%$ & $0.6 \%$ & $1.8 \%$ & $0.8 \%$ & 0.03 \\
\hline & $\mathrm{HF}$ & $0.2 \%$ & $0.1 \%$ & $0.8 \%$ & $0.2 \%$ & $0.3 \%$ & $0.7 \%$ & 0.02 \\
\hline \multicolumn{9}{|c|}{ 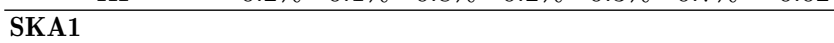 } \\
\hline \multirow[t]{5}{*}{ linear } & $\mathrm{EFT}+c_{\mathrm{S}}$ & $15.8 \%$ & $5.6 \%$ & $28.3 \%$ & $8.4 \%$ & $55.8 \%$ & $94.6 \%$ & 6.1 \\
\hline & EFT+bias & $9.4 \%$ & $13.0 \%$ & $42.7 \%$ & $25.5 \%$ & $63.5 \%$ & $128.5 \%$ & 5.7 \\
\hline & EFT & $6.8 \%$ & $4.1 \%$ & $18.0 \%$ & $5.2 \%$ & $27.1 \%$ & $64.0 \%$ & 3.5 \\
\hline & $\mathrm{HF}+\mathrm{bias}$ & $9.4 \%$ & $13.3 \%$ & $42.7 \%$ & $27.8 \%$ & $73.4 \%$ & $118.4 \%$ & 5.0 \\
\hline & $\mathrm{HF}$ & $5.9 \%$ & $3.3 \%$ & $14.8 \%$ & $4.7 \%$ & $38.8 \%$ & $65.0 \%$ & 4.2 \\
\hline \multirow[t]{5}{*}{ MNL } & $\mathrm{EFT}+c_{\mathrm{s}}$ & $4.1 \%$ & $1.4 \%$ & $11.8 \%$ & $3.8 \%$ & $10.2 \%$ & $14.6 \%$ & 0.8 \\
\hline & $\mathrm{EFT}+$ bias & $3.3 \%$ & $1.4 \%$ & $10.6 \%$ & $4.6 \%$ & $13.5 \%$ & $13.1 \%$ & 0.7 \\
\hline & EFT & $3.2 \%$ & $1.2 \%$ & $10.3 \%$ & $3.2 \%$ & $7.9 \%$ & $12.1 \%$ & 0.6 \\
\hline & $\mathrm{HF}+$ bias & $3.7 \%$ & $1.8 \%$ & $8.8 \%$ & $5.8 \%$ & $15.0 \%$ & $21.5 \%$ & 1.2 \\
\hline & $\mathrm{HF}$ & $2.8 \%$ & $1.3 \%$ & $8.3 \%$ & $3.0 \%$ & $9.7 \%$ & $17.4 \%$ & 1.0 \\
\hline \multicolumn{9}{|l|}{ SKA2 } \\
\hline \multirow[t]{5}{*}{ linear } & $\mathrm{EFT}+c_{\mathrm{S}}$ & $1.4 \%$ & $0.5 \%$ & $3.0 \%$ & $0.8 \%$ & $1.5 \%$ & $7.0 \%$ & 0.2 \\
\hline & $\mathrm{EFT}+$ bias & $1.0 \%$ & $1.1 \%$ & $3.7 \%$ & $2.3 \%$ & $5.8 \%$ & $8.5 \%$ & 0.2 \\
\hline & EFT & $0.6 \%$ & $0.4 \%$ & $1.7 \%$ & $0.6 \%$ & $0.8 \%$ & $3.4 \%$ & 0.1 \\
\hline & $\mathrm{HF}+\mathrm{bias}$ & $0.7 \%$ & $0.8 \%$ & $2.7 \%$ & $1.7 \%$ & $5.0 \%$ & $6.0 \%$ & 0.2 \\
\hline & $\mathrm{HF}$ & $0.5 \%$ & $0.3 \%$ & $1.3 \%$ & $0.4 \%$ & $1.0 \%$ & $4.5 \%$ & 0.2 \\
\hline \multirow[t]{5}{*}{ MNL } & $\mathrm{EFT}+c_{\mathrm{s}}$ & $0.4 \%$ & $0.2 \%$ & $1.3 \%$ & $0.3 \%$ & $0.6 \%$ & $1.3 \%$ & 0.04 \\
\hline & $\mathrm{EFT}+$ bias & $0.3 \%$ & $0.2 \%$ & $1.2 \%$ & $0.5 \%$ & $1.3 \%$ & $1.3 \%$ & 0.03 \\
\hline & EFT & $0.3 \%$ & $0.2 \%$ & $1.1 \%$ & $0.3 \%$ & $0.5 \%$ & $1.0 \%$ & 0.01 \\
\hline & $\mathrm{HF}+\mathrm{bias}$ & $0.3 \%$ & $0.2 \%$ & $0.9 \%$ & $0.5 \%$ & $1.3 \%$ & $1.2 \%$ & 0.03 \\
\hline & $\mathrm{HF}$ & $0.2 \%$ & $0.1 \%$ & $0.8 \%$ & $0.2 \%$ & $0.3 \%$ & $1.0 \%$ & 0.03 \\
\hline
\end{tabular}

TABLE 4

Similar to Table 3 But for a $\left(w_{0}, w_{a}\right)$ CDM scenario. The ERRORS ON $w_{a}$ ARE ABSOLUTE.

the reduced errors but also through the induced correlation between different parameters. We explore this by the correlation matrix $P_{i j}=C_{i j} / \sqrt{C_{i i} C_{j j}}$, where the off-diagonal terms illustrate the degree of correlations. In the case of fully uncorrelated parameters, $\boldsymbol{P}$ would be the identity matrix.

Figures 3 and 4 compare how smaller scales, up to $k_{\max }=0.3 \mathrm{~h} \mathrm{Mpc}^{-1}$, would affect the parameter correlations for HF and EFT, marginalized over $c_{\mathrm{s}}$ and bias parameters respectively, compared to the linear case with $k_{\max }=0.15 h \mathrm{Mpc}^{-1}$. As is evident from the figures, the parameter correlations decrease in most cases as more information becomes available with larger wavenumbers. However, it is also interesting to note that this correlation decrease is not a general rule. With more informative data in hand, the errors on cosmological parameters diminish. However, the degeneracy could increase for some parameters. This could be explained as the following. For these parameters, the large scale imprints are distinguishable, although not tightly constrained. The small scales, on the other hand, yield a lot more, yet not fully distinct, information about the parameters of our interest. Therefor, for these cases, as the errors decrease, the parameter degeneracy increases. 
Figure of merit The overall ability of a particular experiment in constraining the volume spanned by the parameters in the parameter space can be quantitatively described through a figure of merit (FoM). Here we define the FoM as (Albrecht et al. 2006)

$$
\text { FoM }=-\frac{1}{2} \ln (\operatorname{det} C) .
$$

The stronger the constraints on cosmological parameters, the smaller the corresponding volume in the parameter space, and therefor the determinant of $\mathbf{C}$, which leads to bigger FoM. Here we compute FoM as a function $k_{\max }$ with HF and EFT as our theoretical nonlinear prescriptions.

Figure 5 illustrates the FoM in the $\left(w_{0}, w_{a}\right) \mathrm{CDM}$ scenario with different assumptions for the parameter space. The left panel presents the improvement of the FOM in the overall measurement of the equation of state of dark energy as more modes are included in the analysis. The results are marginalized over the rest of cosmological and nuisance parameters. The right panel presents similar results but for the full space of cosmological parameters.

\section{DISCUSSION}

The upcoming large scale surveys will deliver huge amount of information in the mildly nonlinear regime. The analysis of this high precision data requires careful modeling of the nonlinear regime for reliable cosmological inferences. In this work we investigated the bias induced in the parameters when estimated on the basis of the popular Halofit nonlinear model. The bias is most significant for $\sigma_{8}$ and $\Omega_{\mathrm{b}} h^{2}$ with $\sim(4-5) \sigma$, depending on the cosmological scenario, from the galaxy clustering probe of a future Euclid-like survey when modes up to $k_{\text {max }}=0.3 h \mathrm{Mpc}^{-1}$ are included. This clearly demonstrates how the few percent inaccuracy in the nonlinear model translates into a few $\sigma$ bias in the parameter measurements for the near future high precision large scale surveys.

The above analysis was performed with the only free parameters being the cosmologically interesting parameters. A more realistic analysis, however, often includes marginalization over many nuisance parameters. The inclusion of nuisance parameters could increase the estimated uncertainties and reduce the parameter bias. We explored the sensitivity of the forecasted errors, as a function of the maximum wavenumber included in the analysis, to various experimental setups as well as different nonlinear prescriptions and nuisance parameters. In particular we used the EFTofLSS as the main nonlinear model with its so-called sound speeds as the nuisance parameters. We compared the results with those based on Halofit, with the redshift dependence of the galaxy bias assumed unknown and blindly parametrized to be measured by the data itself. The estimated parameter uncertainties from the two recipes are found comparable up to $k_{\max }=0.3 \mathrm{~h} \mathrm{Mpc}^{-1}$. This is evident from the $\mathrm{HF}$ and EFT curves of Figure 5, where the same sets of parameters are included in the analysis with the two nonlinear recipes. It should however be noted that the comparability of the estimated Fisher-based errors simply indicates the similar power of the two approaches in extracting cosmic information from the power spectrum. Obviously, this should not be taken as implying similarity in the predictions of the nonlinear recipes when applied to data, as was seen from biased best-fit measurements of the HF-based analysis.

Relaxing the prior assumptions on the EFT sound speeds and galaxy bias would increase the estimated uncertainty and reduce the parameter biases discussed in Section 2. This is most significant for $\sigma_{8}, \Omega_{\mathrm{c}} h^{2}$ and $w_{a}$ where the estimated errors boost significantly with marginalization over bias parameters at $k_{\max }=$ $0.3 h \mathrm{Mpc}^{-1}$. The nuisance parameters of EFT sound speed are also found to increase the errors (e.g., see the case for $w_{a}$ ), but often to a lesser extent compared to the galaxy bias. A full analysis would include marginalization over both of these nuisance parameter sets. The high impact of these nuisance parameters on the measurement of the cosmologically interesting parameters clearly indicates the need for their appropriate modeling. Tight priors tighten the cosmological constraints and make inferences more concluding. However, if the priors are biased, the final inferences may markedly deviate form the true underlying scenario. A semi-blind parametrization of these nuisance parameters, on the other hand, could significantly boost the errors and hence reduce the parameter bias which could arise due to inappropriate choice of nonlinear prescription. This, in turn, renders the difference between the nonlinear recipes practically less effective.

\section{REFERENCES}

Aghanim, N., et al. 2020, Astron. Astrophys., 641, A6

Albrecht, A., et al. 2006

Alcock, C., \& Paczyński, B. 1979, Nature, 281, 358

Amendola, L., Pettorino, V., Quercellini, C., \& Vollmer, A. 2012, Physical Review D, 85, 103008

Amendola, L., et al. 2018, Living reviews in relativity, 21, 2

Angulo, R., Fasiello, M., Senatore, L., \& Vlah, Z. 2015a, JCAP, 09, 029

Angulo, R. E., Foreman, S., Schmittfull, M., \& Senatore, L. 2015b, JCAP, 10, 039

Assassi, V., Baumann, D., Green, D., \& Zaldarriaga, M. 2014, JCAP, 08,056

Baldauf, T., Mercolli, L., Mirbabayi, M., \& Pajer, E. 2015a, JCAP, 05, 007

Baldauf, T., Mercolli, L., \& Zaldarriaga, M. 2015b, Physical Review D, 92, 123007

Baumann, D., Nicolis, A., Senatore, L., \& Zaldarriaga, M. 2012 JCAP, 07, 051

Bernardeau, F., Colombi, S., Gaztanaga, E., \& Scoccimarro, R. 2002, Physics reports, 367, 1
Blanchard, A., et al. 2019, arXiv preprint arXiv:1910.09273 Bull, P. 2016, Astrophys. J., 817, 26

Carlson, J., White, M., \& Padmanabhan, N. 2009, Physical Review D, 80, 043531

Carrasco, J. J. M., Foreman, S., Green, D., \& Senatore, L. 2014 Journal of Cosmology and Astroparticle Physics, 2014, 057

Carrasco, J. J. M., Hertzberg, M. P., \& Senatore, L. 2012, Journal of High Energy Physics, 2012, 82

Casas, S., Kunz, M., Martinelli, M., \& Pettorino, V. 2017, Physics of the dark universe, 18,73

Chevallier, M., \& Polarski, D. 2001, International Journal of Modern Physics D, 10, 213

Colas, T., D'amico, G., Senatore, L., Zhang, P., \& Beutler, F. 2020, JCAP, 06, 001

Cooray, A., \& Sheth, R. 2002, Physics reports, 372, 1

Crocce, M., \& Scoccimarro, R. 2006, Physical Review D, 73, 063519

D'Amico, G., Gleyzes, J., Kokron, N., Markovic, K., Senatore, L., Zhang, P., Beutler, F., \& Gil-Marín, H. 2020a, JCAP, 05, 005 

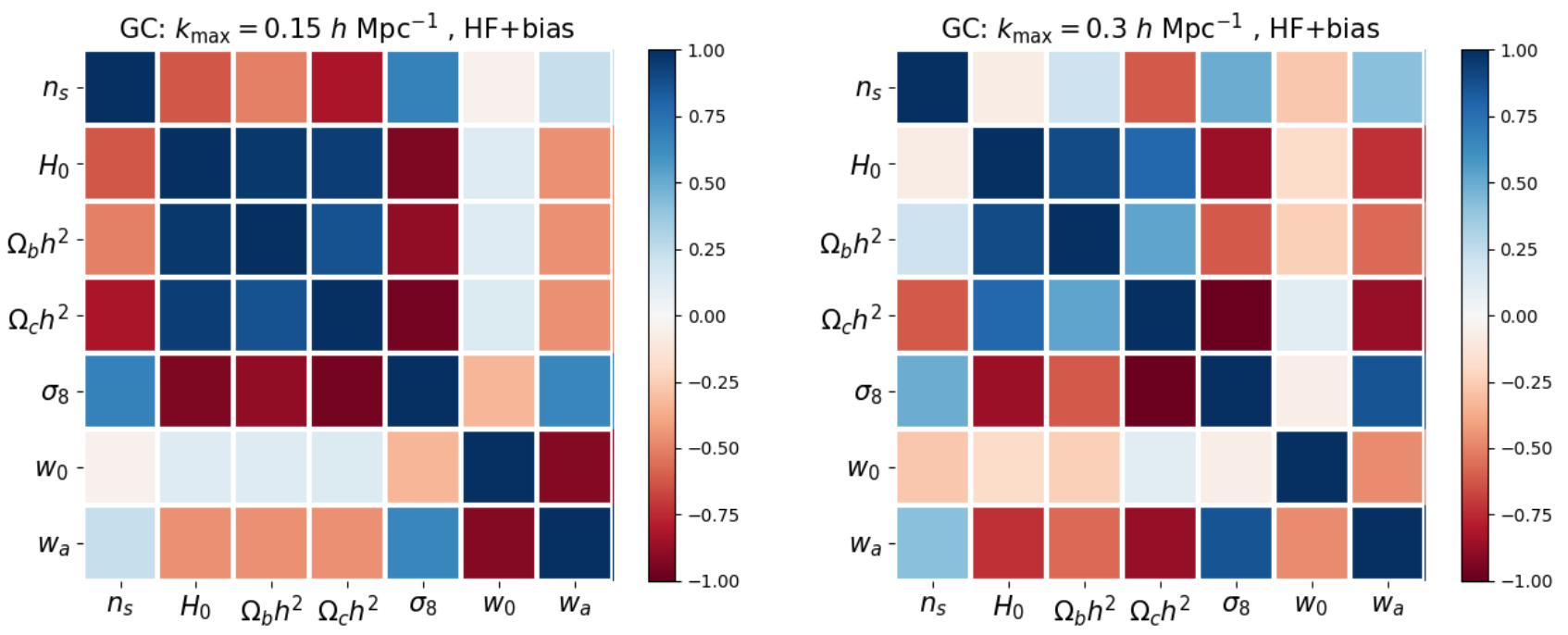

FIG. 3.- Correlation matrix $\boldsymbol{P}$ for the parameters in the $\left(w_{0}, w_{a}\right)$ CDM scenario with the HF nonlinear prescription, in the linear with $k_{\max }=0.15 h \mathrm{Mpc}^{-1}$ (left) and MNL with $k_{\max }=0.3 h \mathrm{Mpc}^{-1}$ (right) regimes.
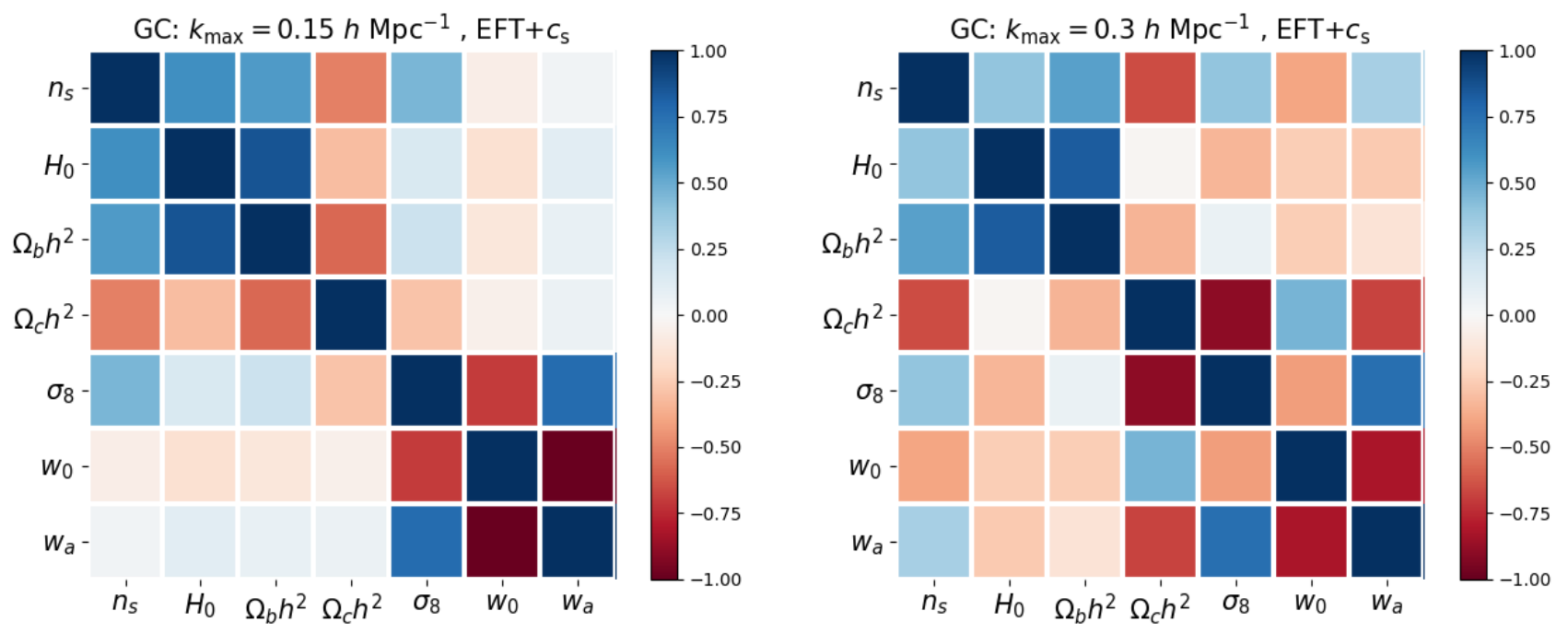

FIG. 4.- Similar to Figure 3 but with EFT nonlinear prescription.
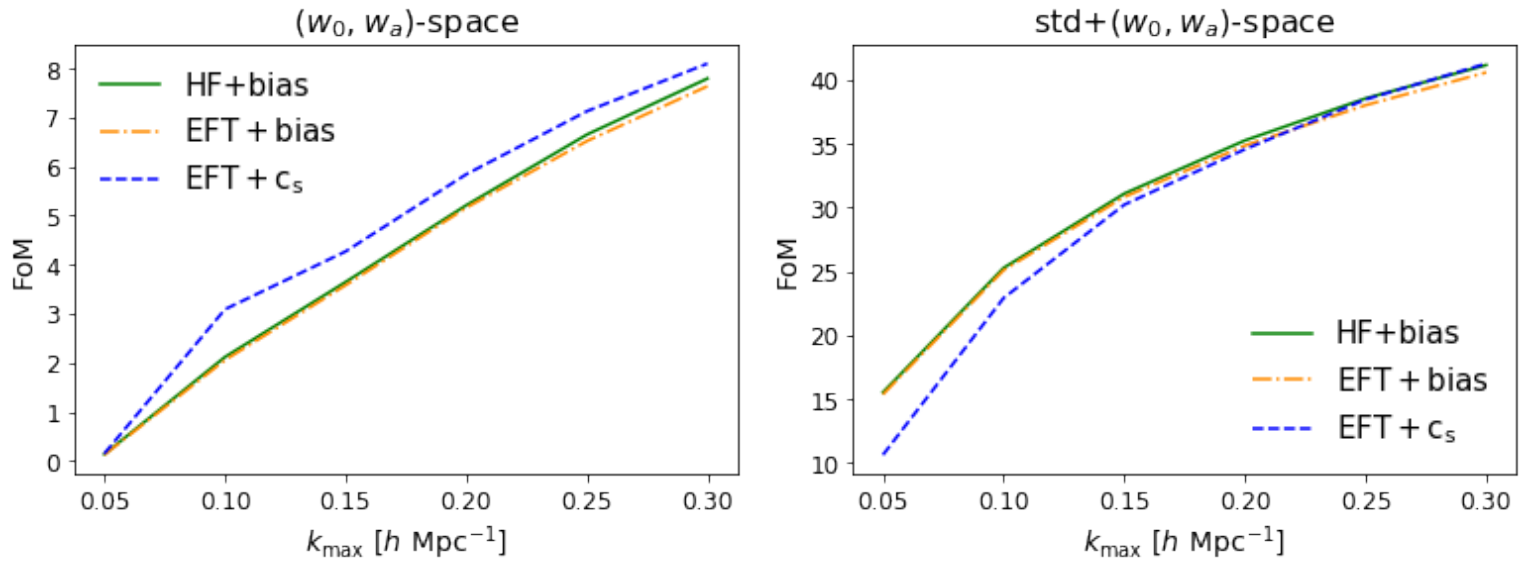

FIG. 5.- The FoM in the $\left(w_{0}, w_{a}\right)$ CDM cosmological model, compared for the HF and EFT nonlinear prescriptions. Left: The FoM for the $\left(w_{0}, w_{a}\right)$ parameter space, marginalized over the cosmological and nuisance parameters. Right: The FoM for the full cosmological parameters, consisting of $\left(w_{0}, w_{a}\right)$ and the standard cosmic parameters, marginalized over the nuisance parameters. 
D'Amico, G., Senatore, L., \& Zhang, P. 2020b, arXiv preprint arXiv:2003.07956

D'Amico, G., Senatore, L., Zhang, P., \& Zheng, H. 2020c, arXiv preprint arXiv:2006.12420

de Putter, R., Doré, O., \& Takada, M. 2013

Di Porto, C., Amendola, L., \& Branchini, E. 2012, Monthly Notices of the Royal Astronomical Society: Letters, 423, L97

Foreman, S., Perrier, H., \& Senatore, L. 2016, Journal of Cosmology and Astroparticle Physics, 2016, 027

Gleyzes, J., Langlois, D., Mancarella, M., \& Vernizzi, F. 2016, Journal of Cosmology and Astroparticle Physics, 2016, 056

Konstandin, T., Porto, R. A., \& Rubira, H. 2019, JCAP, 11, 027

Laureijs, R., et al. 2011, arXiv preprint arXiv:1110.3193

Lewandowski, M., Perko, A., \& Senatore, L. 2015, Journal of Cosmology and Astroparticle Physics, 2015, 019

Lewis, A. 2013, Phys. Rev. D, 87, 103529

Lewis, A., \& Bridle, S. 2002, Phys. Rev. D, 66, 103511

Linder, E. V. 2003, Phys. Rev. Lett., 90, 091301

Ma, C.-P., \& Fry, J. N. 2000, The Astrophysical Journal, 543, 503

Majerotto, E., et al. 2012, Monthly Notices of the Royal Astronomical Society, 424, 1392

Martinelli, M., Calabrese, E., De Bernardis, F., Melchiorri, A., Pagano, L., \& Scaramella, R. 2011, Physical Review D, 83, 023012

Martinelli, M., et al. 2020, arXiv preprint arXiv:2010.12382

Mirbabayi, M., Schmidt, F., \& Zaldarriaga, M. 2015, JCAP, 07, 030
Nishimichi, T., D'Amico, G., Ivanov, M. M., Senatore, L., Simonović, M., Takada, M., Zaldarriaga, M., \& Zhang, P. 2020, arXiv preprint arXiv:2003.08277

Santos, M. G., Alonso, D., Bull, P., Silva, M., \& Yahya, S. 2015

Sartoris, B., et al. 2016, Monthly Notices of the Royal

Astronomical Society, 459, 1764

Seljak, U. 2000, Monthly Notices of the Royal Astronomical Society, 318, 203

Senatore, L. 2015, JCAP, 11, 007

Senatore, L., \& Zaldarriaga, M. 2014

-. 2015, JCAP, 02, 013

Seo, H.-J., \& Eisenstein, D. J. 2007, The Astrophysical Journal, 665,14

Skillman, S. W., Warren, M. S., Turk, M. J., Wechsler, R. H.,

Holz, D. E., \& Sutter, P. 2014, arXiv preprint arXiv:1407.2600

Smith, R. E., et al. 2003, Monthly Notices of the Royal

Astronomical Society, 341, 1311

Sprenger, T., Archidiacono, M., Brinckmann, T., Clesse, S., \& Lesgourgues, J. 2019, Journal of Cosmology and Astroparticle Physics, 2019, 047

Takahashi, R., Sato, M., Nishimichi, T., Taruya, A., \& Oguri, M. 2012, The Astrophysical Journal, 761, 152

Taruya, A., Bernardeau, F., Nishimichi, T., \& Codis, S. 2012, Physical Review D, 86, 103528

Wang, Y. 2012, Monthly Notices of the Royal Astronomical Society, 423, 3631

Wang, Y., Chuang, C.-H., \& Hirata, C. M. 2013, Monthly Notices of the Royal Astronomical Society, 430, 2446 